\title{
Comparative Study of Radius of Curvature of Rounded Edge Hill Obstruction Based on Occultation Distance and ITU-R 526-13 Methods
}

\author{
Mfonobong Charles Uko ${ }^{1}$, Vital Kelechi Onwuzuruike ${ }^{2}$, Eke Godwin Kelechi ${ }^{1}$ \\ ${ }^{1}$ Department of Electrical/Electronic and Computer Engineering, University of Uyo, Uyo, Nigeria \\ ${ }^{2}$ Department of Electrical/Electronic Engineering, Imo State University, Owerri, Nigeria
}

Email address:

promisechibuzor413@yahoo.com (E. G. Kelechi)

\section{To cite this article:}

Mfonobong Charles Uko, Vital Kelechi Onwuzuruike, Eke Godwin Kelechi. Comparative Study of Radius of Curvature of Rounded Edge Hill Obstruction Based on Occultation Distance and ITU-R 526-13 Methods. American Journal of Software Engineering and Applications. Vol. 6, No. 3, 2017, pp. 74-79. doi: 10.11648/j.ajsea.20170603.13

Received: January 29, 2017; Accepted: March 30, 2017; Published: June 12, 2017

\begin{abstract}
In this paper, comparative study of the ITU 526-13 method and the occultation distance-based method for computing the radius of curvature for rounded edged fitted to the vertex of hilltop obstruction is presented. In the study, path profiles of microwave links with isolated single edged hilltop and another path profile with isolated double edged hilltop are used. The frequencies considered are from the $1.5 \mathrm{GHz}$ in the L-band to $36 \mathrm{GHz}$ in the K-band. The result show that for all the frequencies considered, the occultation distance for the single edged hilltop remained constant at $80.923 \mathrm{~m}$ and that for the double edged hilltop remained constant at $532.203 \mathrm{~m}$. Also, while the radius of curvature by the ITU 526-13 method varies with frequency in the two path profiles considered, the radius of curvature by the occultation distance method remained constant for all the frequencies considered in each of the two path profiles considered. Also, for the double edged hilltop, the radius of curvature from ITU 526-13 method greatly exceeded the radius of curvature by the occultation distance method for all the frequencies considered. The least difference in about $58 \%$ at frequency of $1.5 \mathrm{GHz}$ and the difference increased to about $115 \%$ at $36 \mathrm{GHz}$. However, for the single edged hilltop, the radius of curvature for the two methods are relatively equal for frequencies above 6GHz. Essentially, ITU 526-13 method works well like the occultation distance-based method for the single edged hilltop. Further studies are therefore required to determine the situations under which the ITU 526-13 method can be applied in computing the radius of curvature for rounded edge approximation used in diffraction loss computation.
\end{abstract}

Keywords: Radius of Curvature, Rounded Edge Obstruction, ITU 526-13 Method, Occultation Distance, Double Edged Hilltop, Single Edged Hilltop, Fresnel Zone, Radius of Fresnel Zone

\section{Introduction}

In line-of-sight (LOS) microwave communication system significant diffraction loss occurs when obstruction in the signal path extends into the first Fresnel zone [1-8]. Usually, such obstruction can be modeled as either knife edge or rounded edge obstruction [9-16]. Knife edge obstruction model in most cases underestimates the diffraction loss. In that case, the rounded edge model is used for such obstructions as isolated hill [17-19]. The total obstruction for a rounded edge is the sum of the knife edge obstruction and the extra diffraction due to the rounded edge [17-19].

Computation of the extra diffraction due to the rounded edge requires that a rounded edge should be fitted to the vertex of the obstruction. The radius of curvature of the rounded edge is then used to determine the extra diffraction due to the rounded edge. Researchers have developed methods to obtain approximate value for the radius of curvature. One popular method is based on the occultation distance [20-21]. The occultation distance indicates the maximum distance of the shadowed region in the path profile. In the determination of the rounded edge diffraction loss, the occultation distance is given as that distance between the two tangents points near the vertex of the obstruction profile. The first tangent point is made by a line drawn from the transmitter and tangential to the path profile from the transmitter side. The second tangent 
point is made by a line drawn from the receiver and tangential to the path profile from the receiver side. The two tangent line meet at a point above the vertex of the obstruction. The intersection point of the two tangent lines gives the tip of the knife edge. The height of the tip of the knife edge above the LOS line gives the clearance height which is used to compute the knife edge diffraction loss. On the other hand, the distance between the two tangent points on the path profile gives the occultation distance which is used to determine the radius of curvature or the rounded edge fitted to the vertex of the hill obstruction.

Another method of computing the radius of curvature for the rounded edge diffraction obstruction is the ITU-R 526-13 method [22]. In this case, the radius of a parabola fitted to the vertex of the obstruction is determined according to the ITU-R 526-13 formula. The ITU-R 526-13 method requires that the radius of the first Fresnel zone be used to determine the maximum occultation distance that will be considered in the computation of the radius of curvature.

In the occultation distance approach, the tangent is drawn using normal geometric techniques. occultation distance is then determined from the tangent points of the two tangent lines drawn, one from the transmitter and the other one from the receiver. In the ITU-R 526-13 method the radius of the first Fresnel zone is first determined. The radius of curvature is determined based on the points on the obstruction profile that are not more than the radius of the first Fresnel zone from the obstruction apex. Since the radius of the Fresnel zone is a function of frequency, the radius of curvature determined using the ITU-R 526-13 method varies with frequency for the same obstruction. In this paper, the effect of frequency on the radius of curvature by the two methods is studied and their results are compared based on sample rounded edge obstruction in the signal path of a LOS microwave link.

\section{Theoretical Background}

Two methods of computing the radius of curvature of rounded edge fitted to the vertex of isolated hill are compared. The first method used to compute the radius of curvature is the occultation distance based approach whereas the second method is ITU-R 526-13 method [22].

\subsection{Occultation Distance Based Approach}

From the occultation distances the radius, $\mathrm{R}$ of the rounded edge fitted in the vicinity of the hill vertex can be determined as follows [20-21];

$$
R=\frac{2(\mathrm{D})\left(d_{1}\right)\left(d_{2}\right)}{(\alpha)\left[\left(d_{1}\right)^{2}+\left(d_{2}\right)^{2}\right]}
$$

where $\mathrm{D}$ is the occultation distance, $d_{1}$ is distance from the transmitter, $d_{2}$ is distance from the receiver and the angle $\alpha$ in radian is obtained from the graph plot of the path profile and Rounded Edge Diffraction Geometry, as shown in figure 1.

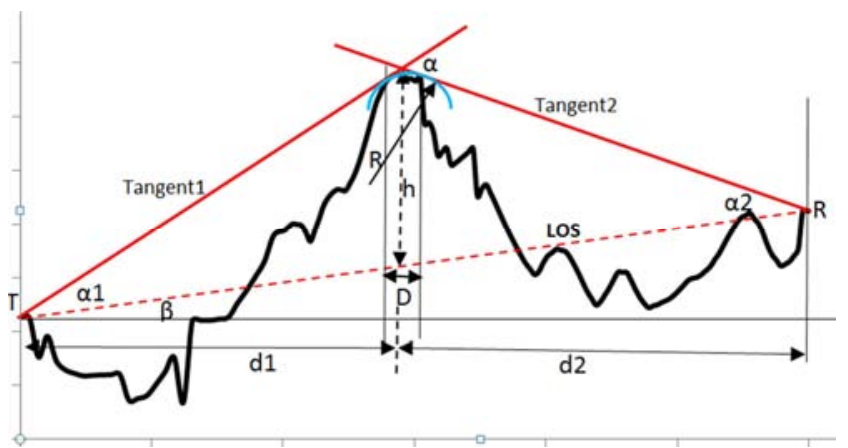

Figure 1. The Path Profile Of The Isolated Hill With The Rounded Edge Diffraction Geometry.

In figure 1, tangent 1 line is drawn from the transmitter to be tangential (at point T1) to the path profile at the vicinity of the hill apex. Also, tangent line is drawn from the receiver to be tangential (at point T2) to the path profile at the vicinity of the hill apex. Let the tangent point of tangent 1 with the path profile be denoted as T2. The occultation distance, D is the point tangent 1 and tangent 2 intersect above the hill vertex, as shown in figure 1.

As shown in figure $2, \mathrm{~d}_{1}$ is distance from the transmitter to the point where tangent 1 and tangent 2 intersect and $d_{2}$ is distance from the receiver to the point where tangent 1 and tangent 2 intersect. Let $\mathrm{d}$ be the distance between the transmitter and the receiver, then;

$$
\mathrm{d}=\mathrm{d}_{1}+\mathrm{d}_{2}
$$

The angle Tangent 1 makes with the LOS is denoted as $\alpha_{1}$ while the angle Tangent 2 makes with the LOS is denoted as $\alpha_{2}$. The angles $\alpha_{1}$ and $\alpha_{2}$ are obtain by cosine rule as follows;

$$
\begin{gathered}
\operatorname{Cos}\left(\alpha_{1}\right)=\frac{\left(\mathrm{S}_{1}\right)^{2}+\left(\mathrm{S}_{3}\right)^{2}-\left(\mathrm{S}_{2}\right)^{2}}{2\left(\mathrm{~S}_{1}\right)\left(\mathrm{S}_{3}\right)} \\
\alpha_{1}=\operatorname{Cos}^{-1}\left(\frac{\left(\mathrm{S}_{1}\right)^{2}+\left(\mathrm{S}_{3}\right)^{2}-\left(\mathrm{S}_{2}\right)^{2}}{2\left(\mathrm{~S}_{1}\right)\left(\mathrm{S}_{3}\right)}\right)
\end{gathered}
$$

Similarly,

$$
\alpha_{2}=\operatorname{Cos}^{-1}\left(\frac{\left(\mathrm{S}_{2}\right)^{2}+\left(\mathrm{S}_{3}\right)^{2}-\left(\mathrm{S}_{1}\right)^{2}}{2\left(\mathrm{~S}_{2}\right)\left(\mathrm{S}_{3}\right)}\right)
$$

where

$S_{1}$ is the length of the tangent 1 measured from the transmitter to the point of intersection of tangent 1 and tangent 2 , as shown in figure 1.

$\mathrm{S}_{2}$ is the length of the tangent 2 measured from the receiver to the point of intersection of tangent 1 and tangent 2 , as shown in figure 1.

$\mathrm{S}_{3}$ is the length of the LOS measured from the transmitter to receiver.

$\mathrm{S}_{1}, \mathrm{~S}_{2}$ and $\mathrm{S}_{3}$ are in meter and they are measured out from the path profile plot and the tangent line drawn on the path profile.The angle $\alpha$ is given as;

$$
\alpha=\alpha_{1}+\alpha_{2}
$$

2.2. The ITU Radius of Curvature Method For The Rounded Edge Diffraction Computation 
In the ITU-R 526-13 method, the radius of curvature of the rounded edge corresponds to the radius of a circle fitted to the apex of a parabola in the vicinity of the top of the obstacle profile. Let $r i$ be the radius of curvature corresponding to the sample $i$ of the vertical profile of the ridge, in figure 2 [22].

$$
r_{i}=\frac{x_{i}{ }^{2}}{2\left(y_{i}\right)}
$$

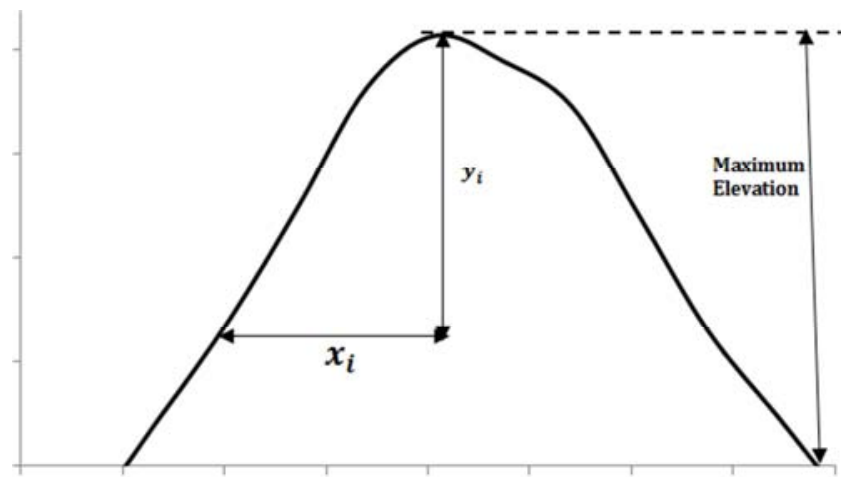

Figure 2. The Geometry of the Vertical Profile of the obstruction used for the determination of the radius of the rounded edge fitted to the vicinity of the obstruction vertex according to ITU Method.

When fitting the parabola, the maximum vertical distance from the apex to be used in this procedure should be of the order of the first Fresnel zone radius where the obstacle is located. As such, in figure 1 and figure 2, the maximum $y_{i}$ is less or equal to the radius of first Fresnel zone at the point of maximum elevation. In the case of $N$ samples, the median radius of curvature of the obstacle is denoted as $R$ where [22]:

$$
R=\sum_{i=1}^{i=N}\left(r_{i}\right)=\sum_{i=1}^{i=N}\left(\frac{x_{i}^{2}}{2\left(y_{i}\right)}\right)
$$

According to the ITU-R 526-13 recommendation, from the obstruction apex (in figure 2) the maximum value of $y_{i}$ should be within the radius of the first Fresnel zone denoted as $\mathrm{r}_{\mathrm{f} 1}$. Essentially;

$$
\operatorname{maximum}\left(y_{i}\right) \leq \mathrm{r}_{\mathrm{f} 1}
$$

The radius of the first Fresnel zone $\left(\mathrm{r}_{1}\right)$ at distance $d_{1}$ from the transmitter and $d_{2}$ from the receiver is given as;

$$
r_{1}=\sqrt{\frac{\left(\kappa\left(d_{1}\right)\left(d_{2}\right)\right)}{\left(d_{1}+d_{2}\right)}}
$$

where $K$ is the signal wavelength which is given as;

$$
\Lambda=\frac{c}{f}
$$

$\mathrm{f}$ is the frequency in $\mathrm{Hz}$ and $\mathrm{c}$ is the speed of light which is $3 \times 10^{8} \mathrm{~m} / \mathrm{s}$.

\section{Results and Discussion}

In the study path profiles of microwave links with isolated single edged hilltop (Table 1) and another path profile with

\begin{tabular}{|c|c|c|c|c|c|c|c|c|c|}
\hline Distance (m) & Elevation (m) & Distance (m) & Elevation (m) & Distance (m) & Elevation (m) & Distance (m) & Elevation (m) & Distance (m) & Elevation (m) \\
\hline 0.0 & 390.9 & 1206.9 & 379.8 & 2413.8 & 401.7 & 3180.8 & 401.8 & 4876.1 & 366.9 \\
\hline 40.2 & 390.9 & 1247.2 & 378.0 & 2454.1 & 397.3 & 3201.0 & 402.7 & 4921.4 & 367.5 \\
\hline 80.5 & 390.9 & 1287.4 & 377.2 & 2494.3 & 401.8 & 3221.2 & 402.5 & 4966.7 & 368.0 \\
\hline 120.7 & 390.7 & 1327.6 & 376.3 & 2534.5 & 403.9 & 3248.1 & 401.7 & 5011.9 & 368.0 \\
\hline 160.9 & 390.7 & 1367.8 & 375.0 & 2574.8 & 401.8 & 3288.3 & 402.0 & 5057.2 & 368.0 \\
\hline 201.2 & 390.9 & 1408.1 & 374.0 & 2615.0 & 402.3 & 3328.6 & 401.8 & 5102.4 & 368.0 \\
\hline 281.6 & 390.4 & 1488.5 & 372.9 & 2695.5 & 408.9 & 3835.2 & 379.8 & 5193.0 & 368.5 \\
\hline 321.8 & 389.6 & 1528.8 & 373.0 & 2735.7 & 411.8 & 3880.4 & 380.3 & 5238.2 & 369.0 \\
\hline 362.1 & 388.4 & 1569.0 & 372.7 & 2755.9 & 410.9 & 3925.7 & 379.3 & 5283.5 & 369.3 \\
\hline 402.3 & 387.5 & 1609.2 & 382.0 & 2776.1 & 411.7 & 3971.0 & 377.9 & 5328.7 & 369.4 \\
\hline 442.5 & 386.9 & 1649.5 & 381.5 & 2796.4 & 412.4 & 4016.2 & 376.9 & 5374.0 & 368.9 \\
\hline 482.8 & 386.7 & 1689.7 & 381.2 & 2816.6 & 411.9 & 4061.5 & 376.0 & 5419.3 & 368.3 \\
\hline 523.0 & 386.7 & 1729.9 & 380.5 & 2836.8 & 412.4 & 4106.7 & 374.9 & 5464.5 & 368.0 \\
\hline 563.2 & 386.4 & 1770.1 & 379.0 & 2857.1 & 412.4 & 4152.0 & 373.5 & 5509.8 & 367.9 \\
\hline 603.5 & 386.1 & 1810.4 & 379.1 & 2877.3 & 412.8 & 4197.2 & 372.4 & 5555.0 & 368.7 \\
\hline 643.7 & 385.6 & 1850.6 & 381.3 & 2897.5 & 411.7 & 4242.5 & 371.5 & 5600.3 & 367.5 \\
\hline 683.9 & 385.2 & 1890.8 & 384.0 & 2917.8 & 410.5 & 4287.8 & 371.2 & 5645.6 & 366.9 \\
\hline 724.2 & 385.1 & 1931.1 & 387.5 & 2938.0 & 411.0 & 4333.0 & 371.1 & 5690.8 & 366.3 \\
\hline 764.4 & 385.4 & 1971.3 & 383.8 & 2958.2 & 409.2 & 4378.3 & 370.7 & 5736.1 & 366.4 \\
\hline 804.6 & 385.5 & 2011.5 & 387.2 & 2978.5 & 398.5 & 4423.5 & 370.2 & 5781.3 & 366.4 \\
\hline 844.8 & 385.6 & 2051.8 & 385.6 & 2998.7 & 399.0 & 4468.8 & 369.4 & 5826.6 & 366.2 \\
\hline 885.1 & 384.6 & 2092.0 & 387.5 & 3018.9 & 398.7 & 4514.1 & 368.3 & 5871.8 & 367.1 \\
\hline 925.3 & 383.4 & 2132.2 & 389.4 & 3039.1 & 398.8 & 4559.3 & 367.6 & 5917.1 & 366.0 \\
\hline 965.5 & 380.9 & 2172.5 & 391.1 & 3059.4 & 398.9 & 4604.6 & 366.7 & 5962.4 & 366.9 \\
\hline
\end{tabular}
isolated double edged hilltop (Table 2) are used. The frequencies considered are from the $1.5 \mathrm{GHz}$ in the L-band to $36 \mathrm{GHz}$ in the Ka-band.

Table 1. The path profiles data for the of microwave links with isolated single edged hilltop. 


\begin{tabular}{|c|c|c|c|c|c|c|c|c|c|}
\hline Distance (m) & Elevation (m) & Distance (m) & Elevation (m) & Distance (m) & Elevation (m) & Distance (m) & Elevation (m) & Distance (m) & Elevation (m) \\
\hline 1005.8 & 377.9 & 2212.7 & 392.8 & 3079.6 & 401.0 & 4649.8 & 366.0 & 6007.6 & 367.7 \\
\hline 1046.0 & 377.0 & 2252.9 & 396.1 & 3099.8 & 400.3 & 4695.1 & 365.5 & 6052.9 & 368.6 \\
\hline 1086.2 & 376.0 & 2293.1 & 396.8 & 3120.1 & 402.2 & 4740.4 & 365.9 & 6098.1 & 370.9 \\
\hline 1126.5 & 375.0 & 2333.4 & 398.3 & 3140.3 & 402.0 & 4785.6 & 366.3 & 6143.4 & 370.9 \\
\hline 1166.7 & 374.1 & 2373.6 & 400.3 & 3160.5 & 401.9 & 4830.9 & 366.5 & 6188.7 & 370.9 \\
\hline
\end{tabular}

Table 2. The path profiles data for the of microwave links with isolated double edged hilltop.

\begin{tabular}{|c|c|c|c|c|c|c|c|c|c|}
\hline Distance (m) & Elevation (m) & Distance (m) & Elevation (m) & Distance (m) & Elevation (m) & Distance (m) & Elevation (m) & Distance (m) & Elevation (m) \\
\hline 0.0 & 390.9 & 1206.9 & 379.8 & 2413.8 & 401.7 & 3180.8 & 411.8 & 4876.1 & 366.9 \\
\hline 40.2 & 390.9 & 1247.2 & 378.0 & 2454.1 & 397.3 & 3201.0 & 412.7 & 4921.4 & 367.5 \\
\hline 80.5 & 390.9 & 1287.4 & 377.2 & 2494.3 & 401.8 & 3221.2 & 412.5 & 4966.7 & 368.0 \\
\hline 160.9 & 390.7 & 1367.8 & 375.0 & 2574.8 & 401.8 & 3288.3 & 412.0 & 5057.2 & 368.0 \\
\hline 201.2 & 390.9 & 1408.1 & 374.0 & 2615.0 & 402.3 & 3328.6 & 411.8 & 5102.4 & 368.0 \\
\hline 241.4 & 390.9 & 1448.3 & 373.4 & 2655.2 & 408.8 & 3368.8 & 407.4 & 5147.7 & 368.0 \\
\hline 321.8 & 389.6 & 1528.8 & 373.0 & 2735.7 & 411.8 & 3880.4 & 380.3 & 5238.2 & 369.0 \\
\hline 362.1 & 388.4 & 1569.0 & 372.7 & 2755.9 & 410.9 & 3925.7 & 379.3 & 5283.5 & 369.3 \\
\hline 402.3 & 387.5 & 1609.2 & 382.0 & 2776.1 & 411.7 & 3971.0 & 377.9 & 5328.7 & 369.4 \\
\hline 442.5 & 386.9 & 1649.5 & 381.5 & 2796.4 & 412.4 & 4016.2 & 376.9 & 5374.0 & 368.9 \\
\hline 482.8 & 386.7 & 1689.7 & 381.2 & 2816.6 & 411.9 & 4061.5 & 376.0 & 5419.3 & 368.3 \\
\hline 523.0 & 386.7 & 1729.9 & 380.5 & 2836.8 & 412.4 & 4106.7 & 374.9 & 5464.5 & 368.0 \\
\hline 563.2 & 386.4 & 1770.1 & 379.0 & 2857.1 & 412.4 & 4152.0 & 373.5 & 5509.8 & 367.9 \\
\hline 603.5 & 386.1 & 1810.4 & 379.1 & 2877.3 & 412.8 & 4197.2 & 372.4 & 5555.0 & 368.7 \\
\hline 683.9 & 385.2 & 1890.8 & 384.0 & 2917.8 & 410.5 & 4287.8 & 371.2 & 5645.6 & 366.9 \\
\hline 724.2 & 385.1 & 1931.1 & 387.5 & 2938.0 & 411.0 & 4333.0 & 371.1 & 5690.8 & 366.3 \\
\hline 764.4 & 385.4 & 1971.3 & 383.8 & 2958.2 & 409.2 & 4378.3 & 370.7 & 5736.1 & 366.4 \\
\hline 804.6 & 385.5 & 2011.5 & 387.2 & 2978.5 & 408.5 & 4423.5 & 370.2 & 5781.3 & 366.4 \\
\hline 844.8 & 385.6 & 2051.8 & 385.6 & 2998.7 & 409.0 & 4468.8 & 369.4 & 5826.6 & 366.2 \\
\hline 885.1 & 384.6 & 2092.0 & 387.5 & 3018.9 & 408.7 & 4514.1 & 368.3 & 5871.8 & 367.1 \\
\hline 925.3 & 383.4 & 2132.2 & 389.4 & 3039.1 & 408.8 & 4559.3 & 367.6 & 5917.1 & 366.0 \\
\hline 965.5 & 380.9 & 2172.5 & 391.1 & 3059.4 & 408.9 & 4604.6 & 366.7 & 5962.4 & 366.9 \\
\hline 1005.8 & 377.9 & 2212.7 & 392.8 & 3079.6 & 411.0 & 4649.8 & 366.0 & 6007.6 & 367.7 \\
\hline 1046.0 & 377.0 & 2252.9 & 396.1 & 3099.8 & 410.3 & 4695.1 & 365.5 & 6052.9 & 368.6 \\
\hline 1086.2 & 376.0 & 2293.1 & 396.8 & 3120.1 & 412.2 & 4740.4 & 365.9 & 6098.1 & 370.9 \\
\hline 1126.5 & 375.0 & 2333.4 & 398.3 & 3140.3 & 412.0 & 4785.6 & 366.3 & 6143.4 & 370.9 \\
\hline 1166.7 & 374.1 & 2373.6 & 400.3 & 3160.5 & 411.9 & 4830.9 & 366.5 & 6188.7 & 370.9 \\
\hline
\end{tabular}

Table 3 and figure 3 show that the for the single edged hilltop, the radius of curvature for the two methods are relatively equal for frequencies above $6 \mathrm{GHz}$. At this frequency $(6 \mathrm{GHz})$ the radius of curvature from ITU 526-13 method is about 3\% above the radius of curvature by the occultation distance method. However, at frequencies above $10 \mathrm{GHz}$, the radius of curvature by the occultation distance method exceeds that from ITU 526-13 method.

Conversely, Table 4 and figure 4 show that the for the double edged hilltop, the radius of curvature from ITU 526-13 method greatly exceeded the radius of curvature by the occultation distance method for all the frequencies considered. The least difference in about $58 \%$ at frequency of $1.5 \mathrm{GHz}$ and the difference increased to about $115 \%$ at $36 \mathrm{GHz}$.

For all the frequencies considered, the occultation distance for the single edged hilltop remained constant at $80.923 \mathrm{~m}$ and that for the double edged hilltop remained constant at 532.203 $\mathrm{m}$. Also, while the radius of curvature by the ITU 526-13 method varies with frequency in each of the two cases considered, the radius of curvature by the occultation distance method remained constant for all the frequencies considered in each of the two path profiles considered. However, there is difference between the radius of curvature by the occultation distance method for the single knife edge and the double knife edge hilltop. The difference is mainly due to the difference in the occultation distance.

Table 3. The radius of curvature in the two methods and the radius of first Fresnel zone for the Single Edged Hilltop for various frequencies.

\begin{tabular}{llllll}
\hline $\mathbf{f}(\mathbf{G H z})$ & $\begin{array}{l}\text { The radius of curvature, } \mathbf{R} \\
(\mathbf{m}) \text { By ITU Method }\end{array}$ & $\begin{array}{l}\text { The radius of curvature R(m) } \\
\text { By Occultation Method }\end{array}$ & $\begin{array}{l}\text { Percentage difference between the radius Radius of First } \\
\text { of curvature in the two method (\%) }\end{array}$ & $\begin{array}{l}\text { Occultation } \\
\text { Fistance (m) }\end{array}$ \\
\hline 1.5 & $5,519.47$ & $3,769.18$ & 46.43663176 & 19.50748 & 80.923 \\
3 & $4,657.10$ & $3,769.18$ & 23.55735235 & 13.79387 & 80.923 \\
6 & $3,888.72$ & $3,769.18$ & 3.171419781 & 9.75374 & 80.923 \\
12 & $3,677.88$ & $3,769.18$ & -2.422449543 & 6.896936 & 80.923 \\
24 & $3,677.88$ & $3,769.18$ & -2.422449543 & 4.87687 & 80.923 \\
36 & $3,677.88$ & $3,769.18$ & -2.422449543 & 3.981948 & 80.923 \\
\hline
\end{tabular}



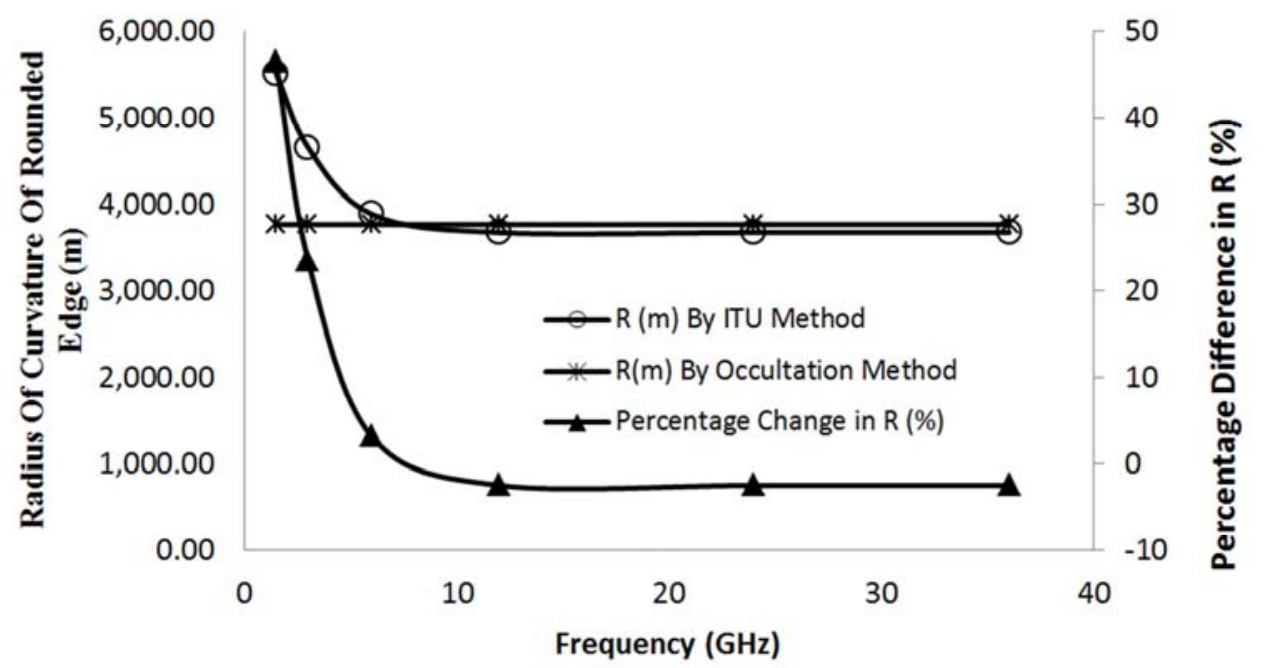

Figure 3. The radius of curvature in the two methods and Percentage difference between the radius of curvature in the two methods for the Single Edged Hilltop for various frequencies.

Table 4. The radius of curvature in the two methods and the radius of first Fresnel zone for the Double Edged Hilltop for various frequencies.

\begin{tabular}{llllll}
\hline $\mathbf{f}(\mathbf{M H z})$ & \multirow{2}{*}{$(\mathbf{m})$ By ITU Method } & \multirow{2}{*}{$\mathbf{R}(\mathbf{m})$ By Occultation Method } & Percentage Difference in R (\%) & $\begin{array}{l}\text { Radius of First } \\
\text { Fresnel Zone (m) }\end{array}$ & $\begin{array}{l}\text { Occultation } \\
\text { Distance (m) }\end{array}$ \\
\hline 1500 & $36,975.97$ & $23,265.15$ & 58.93283487 & 20.05594 & 532.203 \\
3000 & $40,734.97$ & $23,265.15$ & 75.0900696 & 14.18169 & 532.203 \\
6000 & $46,206.97$ & $23,265.15$ & 98.61022159 & 10.02797 & 532.203 \\
12000 & $47,526.22$ & $23,265.15$ & 104.2806893 & 7.090847 & 532.203 \\
24000 & $48,379.92$ & $23,265.15$ & 107.9501214 & 5.013986 & 532.203 \\
36000 & $50,064.92$ & $23,265.15$ & 115.192747 & 4.093902 & 532.203 \\
\hline
\end{tabular}
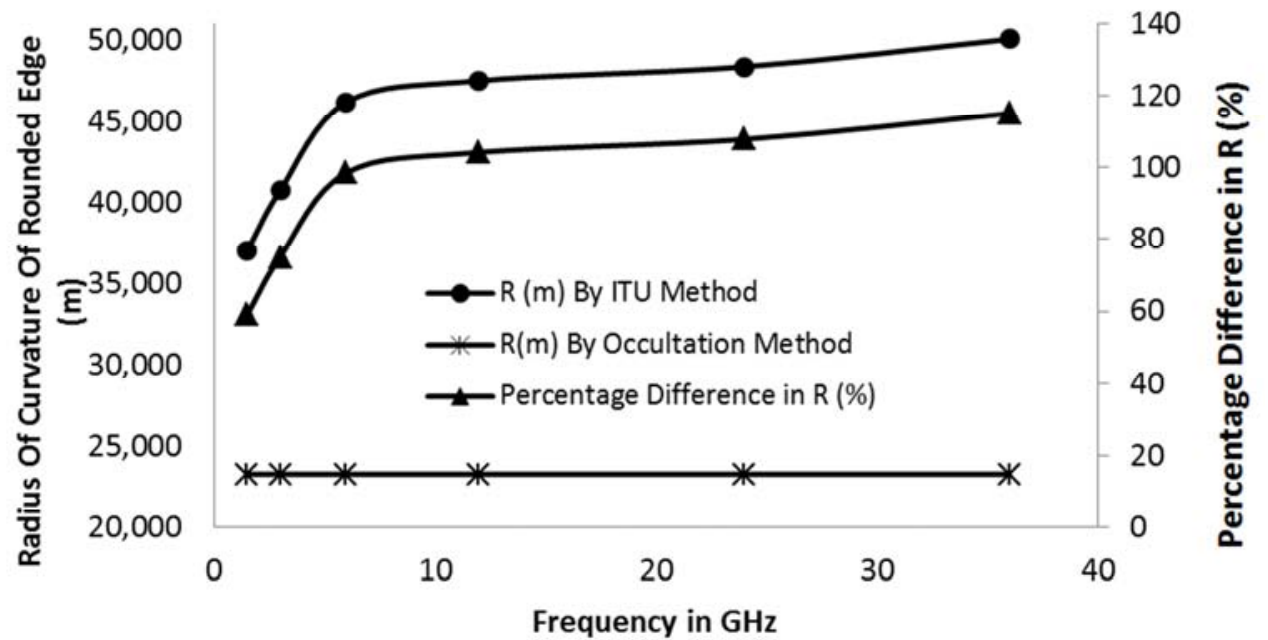

Figure 4. The radius of curvature in the two methods and percentage difference between the radius of curvature in the two method for the double edged hilltop for various frequencies.

\section{Conclusion}

The ITU 526-13 method and the occultation distance-based method for computing the radius of curvature for rounded edged fitted to the vertex of hilltop obstruction is presented. The radius of curvature is usually required for computing rounded edge diffraction loss. The results showed that both frequency and occultation distance affect the radius of curvature computed by the ITU 526-13 method whereas the occultation distance-based method is not affected by frequency of the signal. Also, for small occultation distance and frequencies above $6 \mathrm{GHz}$, the radius of curvature for rounded edged computed by the two methods are relatively equal with minimal difference. However, for large occultation distance the radius of curvature for rounded edged computed by the ITU 526-13 method is very large compared to the values obtained with the distance-based method. Further studies are therefore required to determine the situations under which the ITU 526-13 method can be applied. 


\section{References}

[1] Bassey, D. E., Akpan, A. O., \& Udoeno, E. (2016). UHF Wave Propagation Losses Beyond 40 Percent Fresnel Zone Radius in South-South, Nigeria. International Journal of Science and Research (IJSR), ijsr. net, 5(2), 470-475.

[2] Phillips, C., Sicker, D., \& Grunwald, D. (2013). A survey of wireless path loss prediction and coverage mapping methods. IEEE Communications Surveys \& Tutorials, 15(1), 255-270.

[3] Bassey, D. E., Okoro, R. C., \& Okon, B. E. (2016). Issues Associated with Decimeter Waves Propagation at 0.6, 1.0 and 2.0 Peak Fresnel Zone Levels. International Journal of Science and Research, 5(2), 159-163.

[4] Odedina, P. K., \& Afullo, T. J. (2008). Estimation of Secondary Radioclimatic Variables and Its Application to Terrestrial LOS Link Design in South Africa. AFRICON.

[5] Zennaro, M., Bagula, A., Gascon, D., \& Noveleta, A. B. (2010, August). Planning and deploying long distance wireless sensor networks: The integration of simulation and experimentation. In International Conference on Ad-Hoc Networks and Wireless (pp. 191-204). Springer Berlin Heidelberg.

[6] Green, D. B., \& Obaidat, A. S. (2002). An accurate line of sight propagation performance model for ad-hoc 802.11 wireless LAN (WLAN) devices. In Communications, 2002. ICC 2002. IEEE International Conference on (Vol. 5, pp. 3424-3428). IEEE.

[7] Afullo, T. J., \& Odedina, P. K. (2006). On the K-factor distribution and diffraction fading for southern Africa. Trans. SAIEE, 97(2), 172-181.

[8] Odedina, P. K., \& Afullo, T. J. (2005, July). Effective earth radius factor (k-factor) determination and its application in Southern Africa. In Proceedings of the Second IASTED International Conference on Antennas, Radar and Wave Propagation (pp. 222-227).

[9] Khalid, S. A., Khalsa, A. S., Waitz, I. A., Tan, C. S., Greitzer, E. M., Cumpsty, N. A., \& Marble, F. E. (1998, June). Endwall blockage in axial compressors. In ASME 1998 International Gas Turbine and Aeroengine Congress and Exhibition (pp. V001T01A047-V001T01A047). American Society of Mechanical Engineers.

[10] Qureshi, M. A., Noor, R. M., Shamim, A., Shamshirband, S., \& Choo, K. K. R. (2016). A lightweight radio propagation model for vehicular communication in road tunnels. PloS one, 11(3), e0152727.

[11] Koutitas, G., \& Tzaras, C. (2006). A UTD solution for multiple rounded surfaces. IEEE Transactions on Antennas and Propagation, 54(4), 1277-1283.

[12] Corre, Y., Lostanlen, Y., \& Le Helloco, Y. (2002). A new approach for radio propagation modeling in urban environment: Knife-edge diffraction combined with $2 \mathrm{D}$ ray-tracing. In Vehicular Technology Conference, 2002. VTC Spring 2002. IEEE 55th (Vol. 1, pp. 507-511). IEEE.

[13] Qureshi, M. A., Noor, R. M., Shamim, A., Shamshirband, S., \& Choo, K. K. R. (2016). A lightweight radio propagation model for vehicular communication in road tunnels. PloS one, 11(3), e0152727.

[14] Kumar, K. A. M. (2011). Significance of Empirical and Physical Propagation Models to Calculate the Excess Path Loss. Journal of Engineering Research and Studies.

[15] Qing, L. (2005). GIS Aided Radio Wave Propagation Modeling and Analysis (Doctoral dissertation, Virginia Polytechnic Institute and State University).

[16] Pollock, P. (2001). A Model to Predict Diffraction Attentuation Resulting from Signal Propagation Over Terrain in Low Earth Orbit Satellite Systems (No. AFIT/GSO/ENG/01M-01). AIR FORCE INST OF TECH WRIGHT-PATTERSON AFB OH SCHOOL OF ENGINEERING AND MANAGEMENT.

[17] Östlin, E. (2009). On Radio Wave Propagation Measurements and Modelling for Cellular Mobile Radio Networks (Doctoral dissertation, Blekinge Institute of Technology).

[18] Gálvez, A. M. (2009) Calculation of the coverage area of mobile broadband communications. Focus on land. Master's Thesis Norwegian University of Science and Technology Department of Electronics and Telecommunications.

[19] Pollock, P. (2001). A Model to Predict Diffraction Attentuation Resulting from Signal Propagation Over Terrain in Low Earth Orbit Satellite Systems (No. AFIT/GSO/ENG/01M-01). AIR FORCE INST OF TECH WRIGHT-PATTERSON AFB OH SCHOOL OF ENGINEERING AND MANAGEMENT.

[20] Seybold, J. S. (2005). Introduction to RF propagation. John Wiley \& Sons.

[21] Barué, G. (2008). Microwave engineering: land \& space radiocommunications (Vol. 9). John Wiley \& Sons.

[22] International Telecommunication Union, "Recommendation ITU-R P.526-13: "Propagation by diffraction”, Geneva, 2013. 